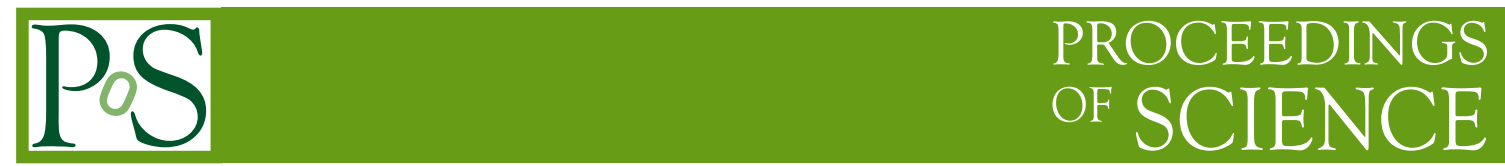

\title{
Searching for new physics in events with three leptons with ATLAS
}

\author{
Katarina Pajchel, on behalf of the ATLAS collaboration* \\ University of Oslo, Norway \\ E-mail: katarina.pajchel@fys.uio.no
}

\begin{abstract}
We investigate the potential of the ATLAS detector to discover new physics events containing three leptons ( $e$ and $\mu$ ) and missing transverse momentum in a hadronically quiet environment. Such final states are predicted in a variety of extensions of the Standard Model. In the context of supersymmetric models, they could result from the direct production of gaugino pairs or from gauginos in decay chains. The gauginos may decay leptonically through real or virtual $W^{ \pm}, Z^{0}$ or sleptons.
\end{abstract}

2008 Physics at LHC

September 29 - 4 October 2008

Split, Croatia

*Speaker. 


\section{Introduction}

In this study [1] we look for events containing three leptons ( $e$ and $\mu)$ and missing transverse momentum in a hadronically quiet environment. Such signal is a clean signature for processes like direct gaugino production. In a scenario where the strongly interacting particles are very heavy and possibly beyond the energy range of the LHC, this could become a SUSY discovery channel.

We use the minimal gravity mediated supersymmetry, mSUGRA, with conserved R-parity as our example signature. The so-called "Focus point region" of the SUSY parameter space is particularly interesting. It is characterised by very heavy squarks and sleptons, while the gauginos are light and dominate the production cross-section. The ATLAS benchmark point SU2 $\left(m_{0}=3550 \mathrm{GeV}\right.$, $m_{1 / 2}=300 \mathrm{GeV}, A_{0}=0, \tan \beta=10, \operatorname{sign} \mu+$ ) representing this region has a next-to-leading-order cross section of $7.18 \mathrm{pb}$.

The main backgrounds are $t \bar{t}, Z b$ and $Z W$. Leptonic decays of $W$ and $Z$ in $t \bar{t}$ and $Z b$ events, both processes with high cross-sections at LHC, can produce two isolated leptons and a third from leptonic b-quark decays. A stringent isolation cut is therefore important in order to reduce them.

\section{Method}

This analysis requires an efficient selection of high quality leptons as well as powerful rejection of leptons from jets and leptonic b-quark decays. Electrons, muons and jets with $p_{T}>10 \mathrm{GeV}$ and within the central barrel region $|\eta|<2.5$ are selected. Lepton isolation cut requires less than $10 \mathrm{GeV}$ of transverse energy deposited in the calorimeter in a cone with $\Delta R=0.2$ around the track $\left(\Delta R \equiv \sqrt{\Delta \eta^{2}+\Delta \phi^{2}}\right)$. Leptons within $\Delta R<0.4$ of a jet are rejected.

Event selection requires at least one pair of opposite sign, same flavour leptons (OSSF) $\left(e^{+} e^{-}\right.$or $\left.\mu^{+} \mu^{-}\right)$and in total three or more leptons, $N_{\ell} \geq 3(\ell \in\{\mathrm{e}, \mu\})$. An extra isolation cut requires $p_{T \text { track,max }}^{\Delta R=0.2}<2 \mathrm{GeV}$ for electrons, $p_{T \text { track,max }}^{\Delta R=0.2}<1 \mathrm{GeV}$ for muons, where $p_{T \text { track,max }}^{\Delta R=0.2}(\ell)$ is the maximum $p_{T}$ of any track in a $\Delta R=0.2$ cone around the lepton. In order to reject backgrounds with a $Z$, OSSF pair invariant mass is required to be outside the range $81.2 \mathrm{GeV}<M_{O S S F}<102.2 \mathrm{GeV}$. The minimum cut of $30 \mathrm{GeV}$ is imposed on $\not p_{T}$. A last cut which is optional requires no jet with $p_{T}>20 \mathrm{GeV}$. It will be referred to as the jet veto.

\section{The discovery potential}

The discovery potential has been studied in terms of signal significance defined as

$$
\mathscr{S}=\frac{S}{\sqrt{S+B}}
$$

where $S$ is the number of signal events and $B$ is the number of background events. It has been studied for two cases: a jet inclusive which applies all event selection cuts except the last jet $p_{T}$ cut and a jet veto case which also includes the jet $p_{T}$ cut. Results:

- The tri-lepton analysis including the jet veto with $10 \mathrm{fb}^{-1}$ :

29 SU2 signal events (all direct gaugino), 210 SM-background events, dominated by $Z W$. Significance: $\mathscr{S}=1.87$, which yields a $5 \sigma$ discovery after $80 \mathrm{fb}^{-1}$. 
- The tri-lepton jet inclusive analysis with $10 \mathrm{fb}^{-1}$ :

177 SU2 signal events (95 of them direct gaugino), 718 SM-background events, dominated by $t \bar{t}$. Significances $\mathscr{S}=5.94$ and $\mathscr{S}=3.34$ are found for the inclusive SUSY and direct gaugino signals respectively.

- The jet inclusive tri-lepton analysis yields a $5 \sigma$ discovery signal after less than $\sim 10 \mathrm{fb}^{-1}$ for all except one studied benchmark point.
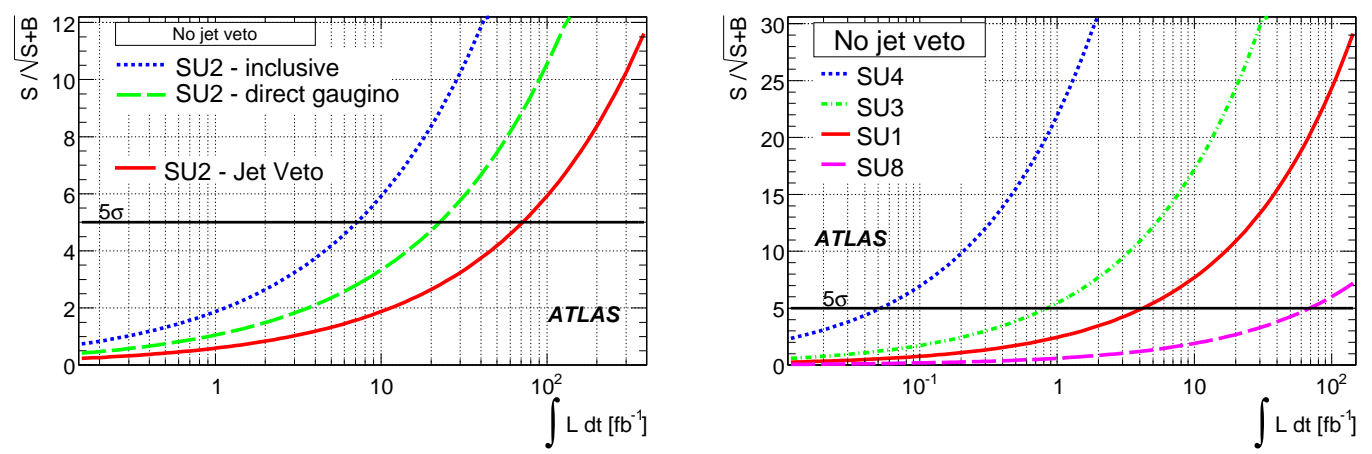

Figure 1: Signal significance $\mathscr{S}$ as function of the integrated luminosity $\int \mathrm{d} t \mathscr{L}$ for (left) SU2, (right) SU1, SU3, SU4, SU8 benchmark points, jet inclusive analysis. The parameters of the SUx points are listed in [1].

Figure 1 shows the discovery potential as a function of the integrated luminosity. Plot (a) summarizes the results obtained for the Focus point benchmark point, while (b) shows that the jet inclusive search reaches a $5 \sigma$ discovery with less than $10 \mathrm{fb}^{-1}$ for several ATLAS benchmark scenarios.

\section{Trigger efficiency and uncertainty}

The trigger efficiency has been studied at the second level (L2) for single, isolated lepton triggers, labeled L2_e22i and L2_20mu. The OR-combination of these two gives a high efficiency ( $>92 \%$ ) as it is expected that at least one of the three leptons will have a relatively high $p_{T}$.

The uncertainty in the SUSY-search including the jet veto will be limited by statistical fluctuations in the background samples $(\sim 6.9 \%)$. The systematic uncertainty is estimated to be $(\sim 3.8 \%)$. In the jet inclusive analysis the statistical uncertainty is smaller $(\sim 3.7 \%)$ while the systematic contribution increases to $\sim 4.9 \%$, caused by the increased fraction of leptons from $b$-quark decays since the top background is much larger without the jet veto.

\section{Conclusions}

The jet inclusive trilepton analysis will yield a $5 \sigma$ discovery signal after less than $\sim 10 \mathrm{fb}^{-1}$ for all except one of the ATLAS benchmark points. To perform a more specific search for direct gaugino production only, a jet veto was also applied; for the benchmark point SU2 this resulted in a signal significance of $\mathscr{S}=1.87$ for $10 \mathrm{fb}^{-1}$ and a $5 \sigma$ discovery after $80 \mathrm{fb}^{-1}$. The significances can be improved by optimising the definitions of the isolation parameters.

\section{References}

[1] ATLAS Collaboration, Expected Performance of the ATLAS Experiment, Detector, Trigger and Physics, to appear, 2008, CERN-OPEN-2008-02, 